\title{
Fatores Associados à Qualidade de Vida de Cuidadores de Idosos em Assistência Domiciliária
}

\section{Factors Associated with the Quality of Life of Caregivers of Elderly Individuals in Home Care}

\author{
Caroline Gomes Ferreira \\ Fisioterapeuta. Especialista em Gerontologia. \\ Endereço: Rua Professor Francisco de Castro, 105, Vila Clementino, \\ CEP 04020-050, São Paulo, SP, Brasil. \\ E-mail: carolgfisioœgmail.com

\section{Tiago da Silva Alexandre} \\ Fisioterapeuta. Doutorando do Programa de Pós-Graduação em \\ Saúde Pública da Faculdade de Saúde Pública da USP. \\ Endereço: Avenida Doutor Arnaldo, 715, Cerqueira César, CEP \\ 01246-904, São Paulo, SP, Brasil. \\ E-mail: tsfisioalexœgmail.com \\ Naira Dutra Lemos \\ Assistente Social. Mestre em Ciências da Saúde. Coordenadora \\ do Programa de Assistência Domiciliária ao Idoso - PADI. Disci- \\ plina de Geriatria e Gerontologia da Escola Paulista de Medicina \\ IUNIFESP. \\ Endereço: Rua Professor Francisco de Castro, 105, Vila Clementino, \\ CEP 04020-050, São Paulo, SP, Brasil. \\ E-mail: nairadutra®uol.com.br
}

\section{Resumo}

O objetivo do presente estudo foi investigar se o comportamento da qualidade de vida (QV) de cuidadores de idosos em assistência domiciliária pode ser influenciado por características sociodemográficas, pela rede de suporte oferecida ao cuidador e por variáveis relacionadas ao ato de cuidar. Foram entrevistados 40 cuidadores de idosos de um Programa de Assistência Domiciliária da cidade de São Paulo. A QV foi mensurada utilizando-se a versão brasileira do Medical Outcomes Study 36 - Item Short-Form Health Survey (SF-36). Características sociodemográficas, as variáveis relacionadas à rede de suporte oferecida ao cuidador e ao ato de cuidar foram obtidas por meio de questionário complementar. A análise de regressão linear mostrou relação independente entre três domínios do $S F-36$ e o maior número de horas dedicadas ao cuidado: domínios capacidade funcional, aspecto físico e aspecto emocional. Possuir mais de oito anos de escolaridade implicou em melhor pontuação no domínio estado geral de saúde e pior pontuação no domínio aspecto social. Os cuidadores com mais de 60 anos de idade apresentaram pior pontuação no domínio aspecto físico e as mulheres pior pontuação no domínio dor. Os filhos ou cônjuges que prestam cuidado aos seus pais ou parceiros apresentaram pior pontuação no domínio aspecto emocional. Os cuidadores que modificaram sua rotina para prestar os cuidados apresentaram pior pontuação no domínio saúde mental. Tanto fatores sociodemográficos como a rede de suporte oferecida ao cuidador e os fatores relacionados à dinâmica do cuidado são capazes de influenciar negativamente a QV de cuidadores principais de idosos em atendimento domiciliário.

Palavras-chave: Assistência Domiciliária; Cuidadores; Qualidade de Vida; Idosos. 


\section{Abstract}

The aim of this study was to investigate whether the quality of life (QOL) of caregivers of elderly individuals in home care is influenced by socio-demographic characteristics, the support network offered to the caregiver and variables related to the act of providing care. Forty caregivers of elderly individuals in a home care program in the city of São Paulo (Brazil) were interviewed. QOL was measured using the Brazilian version of the Medical Outcomes Study 36-Item Short-Form Health Survey (SF-36). Sociodemographic characteristics and variables related to the support network offered to the caregiver and to the act of providing care were obtained through a complementary questionnaire. Linear regression analysis demonstrated an independent relation between greater number of hours dedicated to care and three SF-36 subscales: functional capacity, physical aspects and emotional aspects. Having more than eight years of schooling denoted a better score on the general health state subscale and a worse score on the social aspects subscale. Caregivers over 60 years of age achieved a worse score on the physical aspects subscale and women achieved a worse score on the pain subscale. Sons/daughters or spouses who take care of their parents or partners achieved a worse score on the emotional aspects domain. Caregivers who had changed their routine in order to offer care achieved a worse score on the mental health subscale. Socio-demographic factors, the support network offered to the caregiver, and factors related to the dynamics of care can have a negative influence over the QOL of the main caregivers of elderly individuals in home care.

Keywords: Home Care; Caregivers; Quality of Life; Elderly.

\section{Introdução}

O envelhecimento populacional brasileiro vem ocorrendo, rapidamente, desde 1960, quando as taxas de fecundidade e mortalidade começaram a decair, resultando num crescimento da população com 60 anos ou mais (Cerqueira e Oliveira, 2002). Trata-se de um fenômeno complexo que resulta em mudanças no perfil epidemiológico onde predominam as doenças crônicas não transmissíveis que podem ou não limitar e comprometer a capacidade funcional e a qualidade de vida do idoso culminando em modificações marcantes nos sistemas de cuidados, sejam eles formais ou informais (Trelha e col., 2006; Tang, 2009).

O Brasil possui hoje $8,6 \%$ de sua população constituída por idosos. Estimativas do Instituto Brasileiro de Geografia e Estatística (IBGE) demonstram que esse índice deve alcançar 13\% em 2020 e $20 \%$ em $2050^{1}$.

Diante dessas mudanças demográficas e epidemiológicas, ainda são poucas as respostas do sistema de saúde e de outras políticas setoriais que visem à saúde e ao bem-estar social do idoso e dos seus responsáveis. Dado que a responsabilidade da prestação do cuidado ao idoso pertence à esfera familiar, que cumpre sua norma social ao fazê-lo, a criação do atendimento domiciliário, uma modalidade de assistência capaz de oferecer cuidados ao indivíduo na realidade na qual ele está inserido considerando seus potenciais e suas limitações, foi adotada (Cerqueira e Oliveira, 2002; Rodrigues e Bretas, 2002; Lemos e col., 2006).

Essa modalidade diminui os custos da atenção para a família e para o Estado, reduz o risco de infecções, emprega mais racionalmente os leitos e os recursos hospitalares, além de estimular uma relação médico-paciente mais humanizada (Rehem e Trad, 2005).

$\mathrm{O}$ atendimento domiciliário tem como base a orientação, a informação e o apoio de profissionais especializados em saúde do idoso, sendo dependente do suporte do cuidador para seu bom funcionamento, pois será ele o responsável pela gestão dos cuidados de que o paciente necessita, além de representar

1 FUNDAÇÃO INSTITUTO BRASILEIRO DE GEOGRAFIA E ESTATÍSTICA. Resultado Censo 2ooo. Disponível em <http://www.ibge.gov.br>. Acesso em: 21 mai. 2008. 
a comunicação entre a equipe multiprofissional e o paciente (Amaral e col., 2001; Ricci e col., 2005).

Conceitualmente, o cuidador é definido como aquele que desempenha a função de cuidar de pessoas dependentes numa relação de proximidade física e afetiva, podendo ser um parente, que assume esse papel a partir das relações familiares ou até um profissional, especialmente treinado para tal fim (Wanderley, 1998).

Geralmente a função de cuidador é assumida por uma única pessoa, denominada cuidador principal, seja por instinto, vontade, disponibilidade ou capacidade. Exercer a função de cuidador de idosos dependentes traz uma variedade de efeitos adversos que vão desde dificuldades físicas, sendo a saúde física essencial para o cuidador, até o impacto emocional que esta atividade gera tanto nos familiares cuidadores quanto nos cuidadores formais (Gatz e col., 1991; Cupertino e col., 2006).

A carga relacionada ao cuidado de idosos dependentes faz com que o cuidador mereça atenção tanto quanto o idoso por ele cuidado, pois essa atividade tem impacto em sua saúde, bem-estar e qualidade de vida (Fernandes e Garcia, 2009).

A qualidade de vida representa respostas individuais a fatores físicos (objetivos) e mentais (subjetivos) que contribuem para uma vida "normal", permeada pela satisfação pessoal, autoestima, comparação com o outro, experiências prévias, situação econômica, estado geral de saúde e estado emocional (Farquhar, 1995). É considerada uma percepção eminente humana que abrange múltiplas definições, influenciada por diversos fatores que não se restringem ao tempo, condições socioeconômicas, culturais e de saúde. Embora não exista um significado consensual, há uma concordância acerca do constructo, cuja característica é ser: multidimensio-

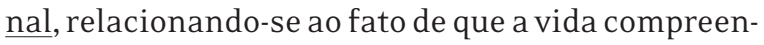
de múltiplas dimensões, tais como a social, mental, material, física, cultural, econômica, dentre outras; dinâmica, dada a sua característica inconstante no tempo e espaço; e subjetiva, determinada pela importância e percepção do significado individual atribuído às experiências inter e intraindividuais (Inouye e col., 2010).

Frente a essa realidade, o desafio das políticas públicas de saúde é criar mecanismos que priorizem as condições de saúde dos idosos, bem como a de seus cuidadores, na grande maioria seus próprios familiares, uma vez que na atualidade não se dispõe de equipamentos sociais capazes de suprir tamanha demanda reprimida (Cerqueira e Oliveira, 2002).

Na tentativa de conhecer a demanda e analisar o impacto que o cuidar representa, o presente estudo tem por objetivo investigar se o comportamento da qualidade de vida (QV) de cuidadores principais de idosos em programa de assistência domiciliária, ligado a uma universidade pública federal, pode ser influenciado por características sociodemográficas, pela rede de suporte oferecida ao cuidador e por variáveis relacionadas ao ato de cuidar.

\section{Método}

Trata-se de uma análise de dados primários de um estudo de natureza seccional realizado em uma população, de conveniência, composta de 40 cuidadores principais de idosos atendidos em um programa de assistência domiciliária na cidade de São Paulo, no período de setembro de 2007 a fevereiro de 2008.

O programa em questão foi fundado em 1999 com o objetivo de atender pacientes idosos cujas condições clínicas e sociais impediam seu acesso ao atendimento ambulatorial de saúde.

No período da pesquisa, estavam inseridos no programa 40 idosos que apresentavam patologia crônica, enfermidade ou condição social que impossibilitasse ou dificultasse a frequência sistemática a um ambulatório, que não estavam dependentes de equipamentos de monitoramento ou suporte à vida, tinham seu domicílio a uma distância máxima da sede do programa de até oito quilômetros e, principalmente, possuíam um cuidador responsável.

As entrevistas com o cuidador principal foram realizadas em São Paulo, através de questionários complementares, após seu consentimento livre e esclarecido, durante a visita domiciliar ao idoso por ele cuidado por um profissional da equipe multidisciplinar que compõe o programa.

O questionário complementar analisou dados sociodemográficos do cuidador, como: faixa etária, sexo, estado civil, escolaridade, situação ocupacional, condição de moradia, renda pessoal, presença de problemas de saúde e vínculo com o paciente; 
informações sobre a rede de suporte do cuidador, como presença de plano de saúde, uso de assistência médica e psicológica e participação em ambulatórios voltados a cuidadores, além de informações relacionadas ao ato de cuidar, como tempo de cuidado ao idoso, horas diárias dedicadas ao cuidado, mudanças de vida para dedicação ao cuidado, presença de outras atividades sob sua responsabilidade além do cuidar e presença de rodízio na prestação de cuidados ao idoso.

No que se refere às atividades realizadas pelos cuidadores para os idosos foram questionados: o preparo e a administração da alimentação, o banho, a higiene íntima, o auxílio na vestimenta, o auxílio na movimentação, a administração da medicação e a realização de curativos. Questionou-se também se o cuidador apresentava dificuldade em realizar alguma das atividades supracitadas.

A qualidade de vida foi mensurada pela versão brasileira do Medical Outcomes Study 36-Item Short-Form Health Survey (SF-36). O SF-36 é um instrumento de qualidade de vida que avalia o perfil da saúde captando aspectos importantes através de oito domínios, sendo de fácil administração e compreensão. É um questionário multidimensional formado por 36 itens, englobados em oito escalas ou componentes: capacidade funcional, aspectos físicos, dor, estado geral da saúde, vitalidade, aspectos sociais, aspectos emocionais e saúde mental. Apresenta um escore final de o a 10o, no qual zero corresponde ao pior escore e cem ao melhor escore, tendo sido utilizado largamente como indicador benéfico para levantamento das condições de saúde e QV em diversas situações e em qualquer população (Ciconelli e col., 1999; Hayes e col., 1995).

Para o tratamento dos dados foi realizada a análise descritiva simples. Testou-se a aderência dos escores dos oito domínios de qualidade de vida à distribuição normal através do teste Shapiro-Wilk e constatou-se que todas as variáveis apresentavam distribuição normal ( $p>0.05$ ).

O grau de associação entre as variáveis quantitativas foi avaliado através da correlação de Pearson. Para testar a diferença de duas médias entre grupos independentes utilizou-se o teste $\mathrm{t}$ - student, e para testar a diferença de três médias entre grupos independentes utilizou-se a ANOVA.
Considerou-se para entrada nos modelos de regressão correlações presentes, positivas ou negativas, consideradas fracas se $=0,3|r|<0,5$; moderadas, se $=0,5|r|<0,7$; e fortes se $|r| 0,7$. Para os testes de diferenças de médias considerou-se para entrada nos modelos de regressão valores de $\mathrm{p} \leq$ 0,05. Em seguida, conduziu-se a análise de regressão linear múltipla, onde a variável vínculo com o idoso foi dividida em subgrupos (dummy-variables) para participarem do modelo estatístico. O nível de significância para a regressão linear múltipla foi fixado em 0,05 .

\section{Resultados}

A amostra apresentou predominância feminina (87,5\%), com média etária de 57,3 anos e desvio padrão (DP) de 13,9. 0 cuidador mais jovem apresentava 26 anos e o mais idoso 82 anos, sendo que 19 dos 40 cuidadores eram idosos. A maior parte da amostra apresentava escolaridade entre 1 e 8 anos (55\%), possuía vida conjugal (65\%), renda mensal de 1 a 3 salários mínimos (62,5\%), era aposentada $(42,5 \%)$ e residia em casa própria $(62,5 \%)$. Desempenhavam papel de cuidador informal $80 \%$ dos entrevistados, sendo que $50 \%$ eram filhos do idoso que recebia os cuidados.

No que se refere à rede de suporte desses cuidadores, 62,5\% deles não possuem plano de saúde, relatando receber atendimento médico somente quando necessário (72,5\%); o mesmo percentual relatou não receber nenhum tipo de atendimento psicológico. Em contrapartida, 65\% da amostra relatou apresentar problemas de saúde. Realizam acompanhamento em Ambulatório de Cuidadores apenas $17,5 \%$ dos cuidadores entrevistados.

O tempo mínimo encontrado dedicado ao cuidado com o idoso foi de 9 meses, e o máximo de 52 anos, apresentando uma mediana de 6 anos. Em relação às horas dedicadas ao cuidar, foi possível verificar que $67,5 \%$ dos cuidadores dedicam-se integralmente ao cuidado (de 18 a 24 horas por dia), seguidos por $12,5 \%$ cuidando do idoso de 6 a 12 horas por dia.

Em relação à mudança na rotina de vida para executar os cuidados, 72,5\% dos cuidadores referem que houve alguma mudança em sua vida, sendo que $25 \%$ relatam que ter deixado de sair foi a mudança mais significativa, acompanhada por deixar de trabalhar (12,5\%). 
Dos 40 cuidadores avaliados, 95\% relataram possuir outras atividades, além do cuidado, sob sua responsabilidade, sendo que $52,5 \%$ relatam cuidar da casa, $17,5 \%$ relatam cuidar da casa e das finanças, $15 \%$ cuidar da casa e da família e 7,5\% cuidar das finanças. Em relação ao rodízio na prestação dos cuidados, 55\% relatam dividir as tarefas com alguém.

A média de atividades executadas para os idosos pelos cuidadores foi de 6,9 com DP de 1,8. Entre as atividades executadas, as mais frequentes, como mostra a Tabela 1, foram: administrar a medicação, preparar a alimentação e dar o banho.

Dentre os cuidadores entrevistados, $52,5 \%$ referiram dificuldades em realizar as atividades para o idoso; a mediana de atividades com dificuldade de execução pelo cuidador foi de 1,o. As atividades que os cuidadores apresentaram maior dificuldade em executar para o idoso, como mostra a Tabela 1, foram auxiliar a movimentação (25\%), dar o banho (20\%), administrar a medicação $(7,5 \%)$ e auxiliar na vestimenta $(7,5 \%)$.

Tabela I - Frequências relativas das atividades executadas pelos cuidadores e seu respectivo relato de dificuldade na execução de 40 cuidadores principais de idosos em atendimento domiciliário na cidade de São Paulo, Sudeste do Brasil, 2007-2008

\begin{tabular}{lcc} 
Atividade Executada & $\begin{array}{c}\text { Frequência relativa da execução (\%) } \\
\text { Administração da medicação }\end{array}$ & $\begin{array}{c}\text { Frequência relativa do relato de } \\
\text { diffuldade na execução (\%) }\end{array}$ \\
Preparo da alimentação & 95 & 2,5 \\
Banho & 92,5 & 5 \\
Auxílio na vestimenta & 90 & 20 \\
Administração da alimentação & 87,5 & 7,5 \\
Higiene íntima & 85 & 7,5 \\
Auxílio na movimentação & 85 & 2,5 \\
Realização de curativos & 85 & 25 \\
\hline
\end{tabular}

De acordo com o questionário de QV SF-36, o domínio em que os cuidadores apresentaram melhor pontuação foi o domínio capacidade funcional com média de 78,2 pontos e desvio padrão de 19,9. A pior pontuação foi no domínio aspecto emocional com média de 50,9 pontos e desvio padrão de 40,5.

As médias do domínio aspecto físico do questionário SF-36 de QV foram diferentes, de forma estatisticamente significativa, no que se refere à faixa etária do cuidador, tendo menor pontuação os cuidadores com idade maior ou igual a 60 anos. Diferenças quanto ao gênero foram observadas no domínio dor, com as mulheres apresentando menor pontuação que os homens.

As médias dos domínios estado geral de saúde e aspecto social do questionário $S F-36$ de QV diferiram, de forma estatisticamente significativa, de acordo com a escolaridade dos cuidadores. Cuida- dores com escolaridade menor que oito anos apresentaram pior pontuação no domínio estado geral de saúde e melhor pontuação no domínio aspecto social quando comparados aos cuidadores com escolaridade maior que oito anos.

As médias dos domínios aspecto físico e aspecto emocional variaram de acordo com o vínculo do cuidador com o idoso. Cônjuges apresentaram as piores pontuações nesses domínios, enquanto os filhos apresentaram as melhores.

As médias do domínio estado geral de saúde variaram de acordo com o tipo de cuidador; curiosamente, os cuidadores formais tiveram menores pontuações que os cuidadores informais.

A Tabela 2 mostra a pontuação média e o desvio padrão dos domínios do $S F-36$ de acordo com as variáveis sociodemográficas. 
Tabela 2 - Pontuação média e desvio padrão dos domínios do SF-36 de acordo com as variáveis sociodemográficas de 40 cuidadores principais de idosos em atendimento domiciliário na cidade de São Paulo, Sudeste do Brasil, 2007-2008

\begin{tabular}{|c|c|c|c|c|c|c|c|c|c|}
\hline \multirow[t]{2}{*}{ Variável Independente } & \multirow[t]{2}{*}{ Categorias } & \multicolumn{8}{|c|}{$\begin{array}{l}\text { Escore médio nos domínios do questionário } \\
\text { SF-36 de acordo com as variáveis sociodemográficas }\end{array}$} \\
\hline & & CF & AF & D & ECS & $\mathrm{v}$ & AS & $\mathrm{AE}$ & $S M$ \\
\hline \multirow[t]{2}{*}{ Faixa etária } & $<60$ anos $n=23$ & 81,9 & $75,0^{*}$ & 54,1 & 70,7 & 50,4 & 63,0 & 53,6 & 59,3 \\
\hline & $\geq 60$ anos $n=17$ & 73,2 & $47,0^{*}$ & 57,2 & 66,7 & 60,0 & 57,6 & 47,2 & 63,0 \\
\hline \multirow[t]{2}{*}{ Sexo } & Feminino $n=35$ & 77,8 & 64,2 & $52,4^{*}$ & 71,4 & 53,5 & 58,0 & 52,4 & 59,5 \\
\hline & Masculino $n=05$ & 81,0 & 55,0 & $76,8^{*}$ & 52,2 & 61,0 & 80,0 & 40,0 & 70,4 \\
\hline \multirow[t]{2}{*}{ Escolaridade } & $<8$ anos $n=22$ & 73,1 & 61,3 & 55,7 & $63,0^{*}$ & 55,0 & $68,4^{*}$ & 54,7 & 60,5 \\
\hline & $\geq 8$ anos $n=18$ & 84,4 & 65,2 & 55,0 & $76,2^{*}$ & 53,8 & $51,3^{*}$ & 46,2 & 61,3 \\
\hline \multirow[t]{3}{*}{ Vínculo } & Cônjuge $n=08$ & 76,8 & $31,2^{*}$ & 58,7 & 61,6 & 51,2 & 59,3 & $12,5^{*}$ & 55,5 \\
\hline & Filhos $n=20$ & 77,7 & $73,7^{*}$ & 57,5 & 73,6 & 52,0 & 61,8 & $61,6^{*}$ & 60,6 \\
\hline & Outros $n=12$ & 80,0 & $66,6^{*}$ & 49,7 & 66,2 & 60,8 & 59,5 & $58,6^{*}$ & 65,0 \\
\hline \multirow[t]{2}{*}{ Tipo de cuidador } & Formal $n=08$ & 78,7 & 71,8 & 52,6 & $55,8^{*}$ & 66,2 & 64,6 & 63,0 & 64,0 \\
\hline & Informal $n=32$ & 78,1 & 60,9 & 56,1 & $72,2^{*}$ & 51,5 & 59,7 & 47,8 & 60,1 \\
\hline Média & & 78,2 & 63,1 & 55,4 & 69,0 & 54,5 & 60,7 & 50,9 & 60,9 \\
\hline Desvio padrão & & 19,9 & 39,2 & 21,8 & 20,7 & 22,4 & 24,7 & 40,5 & 21,5 \\
\hline
\end{tabular}

${ }^{*} p \leq 0,05$

CF - Capacidade Funcional; AF - Aspecto Físico; D - Dor; ECS - Estado Geral de Saúde; V - Vitalidade; AS - Aspecto Social; AE - Aspecto Emocional; SM - Saúde Mental.

As médias do domínio aspecto social do questionário $S F-36$ de QV diferiram, de forma estatisticamente significativa, entre os cuidadores que recebem ou não acompanhamento em ambulatório para cuidadores, tendo aqueles que recebem esse suporte pior pontuação.

A Tabela 3 mostra a pontuação média e o desvio padrão dos domínios do $S F-36$ de acordo com as variáveis relacionadas à rede de suporte.

Observaram-se diferenças, estatisticamente significativas, nas médias dos domínios capacidade funcional, aspecto físico e aspecto emocional no que se refere às horas dedicadas ao cuidado do idoso. Aqueles que dedicam de 12 a 24 horas do dia ao cuidado apresentaram piores pontuações quando comparados àqueles que dedicam de $1 \mathrm{a} 12$ horas do dia ao cuidado.

O escore médio no domínio dor do questionário $S F$-36 de QV apresentou-se menor entre aqueles cuidadores que possuem outras atividades sob sua responsabilidade além do cuidado, diferindo, de for- ma estatisticamente significativa, do escore médio daqueles que não o apresentam.

Os cuidadores que mudaram de rotina para cuidar do idoso apresentaram menor pontuação no domínio saúde mental quando comparados àqueles que não mudaram de rotina; essa diferença de médias foi estatisticamente significativa.

A Tabela 4 mostra a pontuação média e o desvio padrão dos domínios do $S F-36$ de acordo com as variáveis relacionadas ao cuidado.

A análise de regressão linear múltipla mostrou que os cuidadores que dedicam de 12 a 24 horas por dia ao cuidado apresentam 16,59 pontos a menos no domínio capacidade funcional; 30,37 pontos a menos no domínio aspecto físico e 44,27 pontos a menos no domínio aspecto emocional, quando comparados àqueles que dedicam de 1 a 12 horas por dia ao cuidado.

No domínio aspecto físico, a análise demonstrou que cuidadores idosos apresentam 28,48 pontos a menos que cuidadores não idosos. 
Tabela 3 - Pontuação média e desvio padrão dos domínios do SF-36 de acordo com as variáveis relacionadas à rede de suporte de 40 cuidadores principais de idosos em atendimento domiciliário na cidade de São Paulo, Sudeste do Brasil, 2007-2008

\begin{tabular}{|c|c|c|c|c|c|c|c|c|c|}
\hline \multirow{2}{*}{ Variável Independente } & \multirow{2}{*}{ Categorias } & \multicolumn{8}{|c|}{$\begin{array}{l}\text { Escore médio nos domínios do questionário SF-36 } \\
\text { de acordo com as variáveis relacionadas à rede de suporte }\end{array}$} \\
\hline & & CF & AF & D & EGS & V & AS & $\mathrm{AE}$ & SM \\
\hline Recebe atendimento & $\operatorname{sim} n=11$ & 83,6 & 79,5 & 56,7 & 78,0 & 51,8 & 45,4 & 60,6 & 64,0 \\
\hline psicológico & Não $n=29$ & 76,2 & 56,9 & 54,9 & 65,5 & 55,5 & 66,5 & 47,2 & 59,7 \\
\hline Recebe acompanhamento em & $\operatorname{Sim} n=07$ & 79,2 & 64,2 & 54,1 & 76,4 & 40,7 & $41,0^{*}$ & 52,3 & 50,8 \\
\hline ambulatório de cuidadores & Não $n=33$ & 78,0 & 62,8 & 55,7 & 67,4 & 57,4 & $64,9^{*}$ & 50,6 & 63,0 \\
\hline Média & & 78,2 & 63,1 & 55,4 & 69,0 & 54,5 & 60,7 & 50,9 & 60,9 \\
\hline Desvio padrão & & 19,9 & 39,2 & 21,8 & 20,7 & 22,4 & 24,7 & 40,5 & 21,5 \\
\hline
\end{tabular}

${ }^{*} p \leq 0,05$

CF - Capacidade Funcional; AF - Aspecto Físico; D - Dor; ECS - Estado Geral de Saúde; V - Vitalidade; AS - Aspecto Social; AE - Aspecto Emocional; SM - Saúde Mental.

Tabela 4 - Pontuação média e desvio padrão dos domínios do SF-36 de acordo com as variáveis relacionadas ao cuidado de 40 cuidadores principais de idosos em atendimento domiciliário na cidade de São Paulo, Sudeste do Brasil, 2007-2008

\begin{tabular}{|c|c|c|c|c|c|c|c|c|c|}
\hline \multirow[t]{2}{*}{ Variável Independente } & \multirow[t]{2}{*}{ Categorias } & \multicolumn{2}{|c|}{ SF-36 de acordo com as variáveis relacionadas ao cuidado } & & & & & & \\
\hline & & $\mathrm{CF}$ & $\mathrm{AF}$ & D & EGS & V & AS & $\mathrm{AE}$ & SM \\
\hline \multirow[t]{2}{*}{ Horas dedicadas ao cuidado } & $1-12$ horas $n=09$ & $91,1^{*}$ & $86,1^{*}$ & 62,5 & 72,1 & 60,5 & 63,8 & $88,8^{*}$ & 63,5 \\
\hline & $12-24$ horas $n=31$ & $74,5^{*}$ & $56,4^{*}$ & 53,3 & 68,1 & 52,7 & 59,8 & $39,9^{*}$ & 60,1 \\
\hline Outras atividades além do & $\operatorname{sim} n=35$ & 76,8 & 59,2 & $52,5^{*}$ & 68,4 & 54,0 & 59,6 & 47,6 & 59,5 \\
\hline cuidado & Não $n=05$ & 88,0 & 90,0 & $76,0^{*}$ & 72,6 & 58,0 & 68,5 & 74,1 & 70,4 \\
\hline \multirow[t]{2}{*}{ Mudança na rotina para cuidar } & $\operatorname{Sim} n=29$ & 78,6 & 60,3 & 55,4 & 71,4 & 50,6 & 57,7 & 45,9 & $56,8^{*}$ \\
\hline & Não $n=11$ & 77,2 & 70,4 & 55,4 & 62,5 & 64,5 & 68,6 & 64,0 & $71,6^{*}$ \\
\hline Média & & 78,2 & 63,1 & 55,4 & 69,0 & 54,5 & 60,7 & 50,9 & 60,9 \\
\hline Desvio padrão & & 19,9 & 39,2 & 21,8 & 20,7 & 22,4 & 24,7 & 40,5 & 21,5 \\
\hline
\end{tabular}

${ }^{*} p \leq 0,05$

CF - Capacidade Funcional; AF - Aspecto Físico; D - Dor; ECS - Estado Geral de Saúde; V - Vitalidade; AS - Aspecto Social; AE - Aspecto Emocional; SM - Saúde Mental.

Cuidadores do sexo masculino apresentam 24,40 pontos a mais no domínio dor quando comparados às cuidadoras do sexo feminino. A escolaridade maior ou igual a oito anos permitiu que os cuidadores apresentassem 13,23 pontos a mais no domínio estado geral de saúde e 17,38 pontos a menos no domínio aspecto social, quando comparados àqueles com escolaridade inferior a oito anos.

Os cuidadores que não recebem acompanhamento em ambulatório apresentaram 24,30 pontos a mais no domínio aspecto social, quando comparados com aqueles que recebem esse auxílio. Os que não apresentaram vínculo familiar com o idoso obtiveram 15,65 pontos a mais no domínio aspecto emocional, quando comparados àqueles que apresentaram; e aqueles cuidadores que não mudaram sua rotina para prestar o cuidado obtiveram 14,80 pontos a mais no domínio saúde mental quando comparados àqueles que mudaram sua rotina. Todos os dados da análise de regressão linear múltipla são mostrados na Tabela 5 . 
Tabela 5 - Modelo final da análise de regressão linear para cada domínio de qualidade de vida em 40 cuidadores principais de idosos em atendimento domiciliário na cidade de São Paulo, Sudeste do Brasil, 2007-2008. $(\mathrm{N}=40)$

\begin{tabular}{|c|c|c|c|}
\hline \multirow{2}{*}{$\begin{array}{l}\text { Variáveis } \\
\text { Domínio }\end{array}$} & \multicolumn{3}{|c|}{ Modelo Final } \\
\hline & Coeficiente B & Erro padrão & $p$ \\
\hline \multicolumn{4}{|l|}{ Capacidade Funcional } \\
\hline Horas dedicadas ao cuidado ( $12-24$ horas $)$ & $-16,59$ & 7,16 & $0,026^{*}$ \\
\hline \multicolumn{4}{|l|}{ Aspecto Físico } \\
\hline Faixa Etária ( $\geq 60$ anos) & $-28,48$ & 11,27 & $0,016^{*}$ \\
\hline Horas dedicadas ao cuidado ( $12-24$ horas) & $-30,37$ & 13,34 & $0,029^{*}$ \\
\hline \multicolumn{4}{|l|}{ Dor } \\
\hline Sexo (Masculino) & 24,40 & 9,8 & $0,017^{*}$ \\
\hline \multicolumn{4}{|l|}{ Estado Geral de Saúde } \\
\hline Escolaridade ( $\geq 8$ anos) & 13,23 & 6,32 & $0,043^{*}$ \\
\hline \multicolumn{4}{|l|}{ Aspecto Social } \\
\hline Escolaridade ( $\geq 8$ anos) & $-17,38$ & 6,93 & $0,017^{*}$ \\
\hline Não recebe acompanhamento em ambulatório de cuidadores & 24,30 & 9,08 & $0,011^{*}$ \\
\hline \multicolumn{4}{|l|}{ Aspecto Emocional } \\
\hline Não tem vínculo familiar com o idoso & 15,65 & 7,81 & $0,05^{*}$ \\
\hline Horas dedicadas ao cuidado ( $12-24$ horas) & $-44,27$ & 13,10 & $0,002^{*}$ \\
\hline \multicolumn{4}{|l|}{ Saúde Mental } \\
\hline Não mudou a rotina para prestar o cuidado & 14,80 & 7,35 & $0,05^{*}$ \\
\hline
\end{tabular}

$* p \leq 0,05$

\section{Discussão}

A predominância feminina e a idade média de 57,3 anos da amostra estudada confirmam dados de pesquisas envolvendo cuidadores de idosos e reforçam o papel da mulher na sociedade, onde desde muito jovem já lhe é imposto o dever de organizar a casa e a família, restando-lhe a tarefa de cuidar de seus membros mais idosos (Garrido e Menezes, 2004; Ekwall e col., 2004; Lemos e col., 2006; Chou e col., 2007; Vickrey e col., 2009; Pimenta e col., 2009).

Apesar de a maioria dos cuidadores principais de idosos no presente estudo serem filhos, uma parte era constituída por cônjuges. Dentre os cônjuges, é importante ressaltar o fato de encontrarmos uma realidade de um idoso cuidando de outro idoso mais dependente. A semelhança de faixa etária existente nessa relação de cuidado é capaz de influenciar aspectos físicos, emocionais e sociais do cuidador contribuindo diretamente para seu isolamento social (Caldas, 2004; Ekwall e col., 2004; Pimenta e col., 2009; Vickrey e col., 2009).
Várias pesquisas têm sido realizadas para verificar a sobrecarga do cuidado relacionando aspectos físicos, psicológicos, financeiros e qualidade de vida (Tomomitsu e col., 2010). Cuidadores idosos apresentam perdas funcionais que repercutem numa diminuição de suas potencialidades globais, o que pode explicar a relação existente entre o domínio capacidade funcional e a faixa etária superior a 60 anos.

Neste estudo, o pior desempenho dos cônjuges nos domínios aspecto físico e emocional, quando comparados aos filhos, contradiz os relatos de Trelha e colaboradores (2006), que demonstram que cuidadores jovens sofrem maior impacto da atividade de cuidar em sua qualidade de vida, pois muitas vezes deixam sua vida profissional e social de lado para exercer tal atividade, mesmo que por iniciativa própria, mas vem ao encontro dos dados de Conde-Sala e colaboradores (2009).

Lemos e colaboradores (2006), em seu estudo com cuidadores de pacientes demenciados, também 
encontraram cônjuges com pior desempenho no domínio emocional de qualidade de vida, assim como Conde-Sala e colaboradores (2009) demonstraram que é o cônjuge cuidador quem sofre o maior impacto da doença de quem ele cuida.

Um dado que chama a atenção é o pior desempenho dos cuidadores formais no domínio estado geral de saúde quando comparados aos cuidadores informais, pois demonstra que inclusive os cuidadores contratados podem não estar preparados para enfrentar as dificuldades do cuidar, tornando então o problema do cuidado mais grave do que aparenta. Esse dado é coincidente com os de Takai e colaboradores (2009), que relataram que o cuidador formal sofre sobrecarga física e mental com implicações negativas à sua saúde.

Um dado que complementa o supracitado é a existência de correlação entre a escolaridade e o domínio estado geral de saúde. Os indivíduos que possuem maior escolaridade apresentaram maior pontuação nesse domínio. A escolaridade apresentou também correlação, porém inversa, com o domínio aspecto social, pois indivíduos com maior escolaridade são capazes de perceber a restrição na manutenção de sua vida social em decorrência dos cuidados prestados ao idoso e, consequentemente, apresentam maior impacto subjetivo da doença em sua QV (Lemos e col., 2006).

Cuidadores que recebem acompanhamento psicológico no Ambulatório de Cuidadores apresentaram pior desempenho no domínio aspecto social do $S F-36$ quando comparados com aqueles que não o recebem. Esse fato está associado ao estresse passado pelo cuidador na prestação do cuidado, gerando problemas emocionais, físicos, interpessoais e ocupacionais, fazendo com que esse cuidador precise de cuidados emergenciais, diferente daqueles que não buscaram o serviço por ainda conseguirem controlar suas demandas emocionais, interpessoais e ocupacionais (Brogna, 1998).

Por esse motivo, é importante desenvolver programas destinados a prevenir esses efeitos e a trabalhar a busca da QV dos cuidadores, pois aqueles que não contam com apoio social formal ou informal têm maior probabilidade de desenvolver problemas de saúde física e psíquica, quando comparados aos que se sentem queridos, respeitados e apoiados de alguma forma (Tang, 2009; Cerqueira e Oliveira, 2002; Néri e col., 2002).

The National Survey of families and households (NSFH) Health and human services demonstra que os cuidadores de pessoas com 50 anos ou mais gastam em média 17,9 horas por semana prestando cuidados. Esse tempo aumenta para 20 horas quando os idosos são maiores de 65 anos. Já o estudo Family care giving in the US findings from a National Survey informa que entre aqueles cuidadores de familiares ou amigos maiores de 50 anos, aproximadamente 20\% (4,5 milhões entre 22,4 milhões) gastam mais de 40 horas por semana em cuidados (Leme, 2006). Esses cuidados expõem o cuidador, continuamente, a eventos estressantes alterando gradualmente seu bem-estar geral e sua qualidade de vida (Fernandes e Garcia, 2009).

Outro ponto importante com impacto na qualidade de vida do cuidador são as mudanças na própria vida que este tem de fazer para prestar o cuidado ao idoso. Ter realizado mudanças na rotina de vida para executar os cuidados apresentou correlação com o domínio saúde mental de QV dos cuidadores entrevistados. Isso se deve à reestruturação da vida dessas pessoas, como descrito por Cattani e Girardon-Perlini (2004), que relatam que o cuidar gera a necessidade de o indivíduo que cuida abdicar-se de algumas atividades que eram praticadas habitualmente, negligenciando sua própria vida ou parte dela.

Um dos maiores obstáculos apontados enquanto vivenciam o processo de cuidar é a impossibilidade de sair de casa, de realizar atividades de lazer, pois, de forma geral, ficam atrelados à responsabilidade e à preocupação diária com a doença e o cuidado do idoso. Cuidar de uma pessoa dependente faz com que o estilo de vida do cuidador seja modificado em função das necessidades do outro. Independentemente do fato de o cuidador ser uma pessoa jovem ou idosa, suas atividades de recreação e convívio social acabam sendo alteradas e dando a este a sensação de não ter autonomia para gerenciar a própria vida e ter de viver em torno do outro. Esses dados corroboram com Fernandes e Garcia (2009), que relatam que apesar de estarem disponíveis para servir, os cuidadores encontravam-se cansados, com falta de tempo para si e com escassez de suporte familiar. 
Apresentar outras atividades sob sua responsabilidade acarretou em pior pontuação no domínio dor do SF-36. Esses dados, segundo Karsch (1998), parecem ser frequentes, pois as atividades relacionadas ao lar estão inscritas nas necessidades de tarefas desenvolvidas pelos cuidadores que se estendem ao longo do dia sem contar com o auxílio de terceiros. Conciliar as atividades domésticas com os cuidados pessoais e os cuidados ao idoso dependente demanda habilidade na organização do tempo e no cumprimento dessas tarefas. Esses dados são corroborados por Gonçalves e colaboradores (2006), que relatam que aqueles que possuem outras atribuições, além dos cuidados referentes aos idosos, são mais predispostos ao estresse, isolamento social, depressão, fadiga e risco de adoecimento. 0 sexo masculino apresentou melhor pontuação no domínio dor quando comparado ao sexo feminino, dados que podem ser justificados pela diferença na construção da experiência de adoecimento tanto na percepção quanto no relato diferenciado entre os sexos, como relatado por Tang (2009).

Os cuidadores sentem-se sobrecarregados pela demanda de cuidados, por terem de realizar tarefas pessoais do idoso, como banho, higiene, sendo caracterizadas por atividades absorventes, que preenchem o dia e, às vezes, a noite da pessoa que assume esses cuidados, intensificando sua imersão na função de cuidar e expondo o cuidador às consequências negativas dessa atividade, elevando mais o seu nível de tensão (Cattani e Girardon-Perlini, 2004; Fernandes e Garcia, 2009).

As mudanças que ocorrem na vida do cuidador relacionam-se principalmente à reformulação da rotina, preparação da alimentação, administração da medicação, higiene pessoal, vestimenta, locomoção, transferências, entre outras tarefas, as quais podem apresentar-se de forma árdua e desgastante (Karsch, 1998). Esses dados são semelhantes ao nosso estudo, em que os cuidadores relataram dificuldades para executar algumas das tarefas pessoais para o idoso; estas apresentaram associação com os domínios dor, aspecto social, emocional e estado geral de saúde.

Ricci e colaboradores (2005) e Fernandes e Garcia (2009) relatam que os cuidadores apresentam maiores dificuldades em relação à execução da higiene pessoal e ao cuidado com ferimentos, pois muitas vezes esses idosos outrora foram maridos autoritários, que jamais aceitariam perder sua autonomia e, hoje, dependentes, por vezes sentindo-se invadidos em sua privacidade, apresentam forte tensão. Estes achados diferem do presente estudo no qual as maiores dificuldades relatadas foram auxiliar a movimentação e o banho, seguidas de administrar alimentação e auxiliar na vestimenta. Esses dados podem ser confirmados por Perlini e Faro (2005), que relatam que atividades que necessitem de esforço físico certamente são as que causam maior dificuldade para o cuidador. Dessa forma, para o cuidado de um idoso dependente é necessário ter condicionamento físico suficiente para a execução de tarefas pesadas. A realização da higiene corporal do idoso demanda esforço físico por parte do cuidador, além de organização e intimidade, independentemente de qual é sua dependência.

É considerada uma limitação do estudo o fato de a amostra ser constituída por conveniência, o que limita a expansão dos resultados para a população de cuidadores de idosos em geral. É importante ressaltar que estudos transversais estão sujeitos ao viés de causalidade reversa, pois a exposição e os fatores são coletados num mesmo momento, prejudicando a análise de associações causais; dessa forma, os resultados encontrados devem ser apreciados levando, também, esse fator em consideração.

\section{Considerações Finais}

$\mathrm{Na}$ amostra estudada, a maioria dos cuidadores é do sexo feminino, filhos e com baixa escolaridade. Esses cuidadores possuem problemas de saúde, dedicam-se integralmente ao cuidado, mudaram sua rotina para exercer essa função, realizam muitas atividades para o idoso de que cuidam e relatam dificuldade em fazê-las.

Os cuidadores do sexo feminino, com mais de 60 anos, filhos ou cônjuges do idoso a receber os cuidados, que dedicam muitas horas do dia ao cuidado, que mudaram sua rotina para prestar o cuidado e que recebem acompanhamento em ambulatório para cuidadores são os que apresentam pior percepção da qualidade de vida na amostra estudada. 


\section{Referências}

AMARAL, N. N. et al. Assistência domiciliar à saúde (home health care): sua história e relevância para o sistema de saúde atual. Revista de Neurociências, São Paulo, v. 9, n. 3, p. 111-117, 2001.

BROGNA, L. Caring for caregivers: home care nursing's challenge. In: ALLENDER, J. A.; RECTOR, C. L. (Org.). Readings in gerontological nursing. Philadelphia: Lippincot, 1998. p. 515-522.

CALDAS, C. P. Cuidado familiar: a importância da família na atenção à saúde do idoso. In: SALDANHA, A. L.; CALDAS, C. P. (Org.). Saúde do idoso: a arte de cuidar. Rio de Janeiro: Interciência, 2004. p. 4-7.

CATTANI, R. B.; GIRARDON-PERLINI, N. M. O cuidar do idoso doente no domicílio na voz de cuidadores familiares. Revista Eletrônica de Enfermagem, Goiânia, v. 6, n. 2, p. 254-271, 2004.

CERQUEIRA, A.; OLIVEIRA, N. I. L. Programa de apoio a cuidadores: uma ação terapêutica e preventiva na atenção à saúde dos idosos. Psicologia, São Paulo, v. 13, n. 1, p. 133-150, 2002.

CHOU, Y. C. et al. The quality of life of family caregivers of adults with intellectual disabilities in Taiwan. Journal of Applied Research in Intellectual Disabilities, Lancashire, v. 20, p. 200210, 2007.

CICONELLI, R. M. et al. Tradução para a língua portuguesa e validação do questionário genérico de avaliação de qualidade de vida SF-36 (Brasil SF-36). Revista Brasileira de Reumatologia, São Paulo, v. 39, n. 3, p. 143-150, 1999.

CONDE-SALA, J. L. et al. Factors related to perceived quality of life in patients with Alzheimer's disease: the patient's perception compared with that of caregivers. International Journal of Geriatric Psychiatry, Manchester, v. 24, n. 6, p. 585-594, 2009.

CUPERTINO, A. P. F. B.; ALDWIN, C. M.; OLIVEIRA, B. H. D. Moderadores dos efeitos do estresse na saúde auto-percebida de cuidadores. Interação em Psicologia, Curitiba, v. 10, n. 1, p. 9-18, 2006.
EKWALL, A.; SIVBERG, B.; HALLBERG, I. R. Dimensions of informal care and quality of life among elderly family caregivers. Scandinavian Journal of Caring Science, Oxford, v. 18, p. 239-248, 2004.

FARQUHAR, M. Definitions of quality of life; a taxonomy. Journal of Advanced Nursing, Oxford, v. 22, p. 502-508, 1995.

FERNANDES, M. G. M.; GARCIA, T. R.

Determinantes da tensão do cuidador familiar de idosos dependentes. Revista Brasileira de Enfermagem, Brasília, DF, v. 62, n. 3, p. 393-399, 2009.

GARRIDO, R.; MENEZES, P. R. Impacto em cuidadores de idosos com demência atendidos em um serviço psicogeriátrico. Revista de Saúde Pública, São Paulo, v. 38, n. 6, p. 835-841, 2004. GATZ, M.; BENGSTON, V. L.; BLUM, M. G. Caregiving families. Handbook of the psycology of aging, San Diego, v. 3, p. 405-426, 1991.

GONÇALVES, L. H. T. et al. Perfil da família cuidadora de idoso doente/fragilizado do contexto sociocultural de Florianópolis, SC. Texto Contexto Enfermagem, Florianópolis, v. 15, n. 4, p. 570-577, 2006.

HAYES, V. et al. The SF-36 health survey questionnaire is it suitable for use with older adults? Age and Ageing, Oxford, v. 24, n. 2, p. 120$125,1995$.

INOUYE, K.; PEDRAZZANI, E. S.; PAVARINI, S. C. I. Implicações da doença de Alzheimer na qualidade de vida do cuidador: um estudo comparativo. Cadernos de Saúde Pública, Rio de Janeiro, v. 26, n. 5, p. 891-899, 2010.

KARSCH, U. M. S. Envelhecimento com dependência: revelando cuidadores. São Paulo: EDUC, 1998.

LEME, M. D. Treinamento de cuidadores de idosos: impacto na sua qualidade de vida e saúde. 2006. Dissertação (Mestrado em Ciências)-Faculdade de Medicina da Universidade de São Paulo, São Paulo, 2006. 
LEMOS, N. D.; GAZZOLA, J. M.; RAMOS, L. R. Cuidando do paciente com Alzheimer: o impacto da doença no cuidador. Saúde e Sociedade, São Paulo, v. 15, n. 3, p. 170-179, 2006.

NÉRI, A. L. E.; CARVALHO, V. A. M. L. O bem estar do cuidador: aspectos psicossociais. In:

FREITAS, E. V. et al. (Org.). Tratado de geriatria e gerontologia. Rio de Janeiro: Guanabara Koogan, 2002. p. 778-79o.

PIMENTA, G. M. F. et al. Perfil do familiar cuidador de idoso fragilizado em convívio doméstico da grande Região do Porto, Portugal. Revista da Escola de Enfermagem da USP, São Paulo, v. 43, n. 3, p. 609-614, 2009.

PERLINI, N. M. O. G.; FARO, A. C. M. Cuidar de pessoa incapacitada por acidente vascular cerebral no domicílio: o fazer do cuidador familiar. Revista da Escola de Enfermagem da USP, São Paulo, v. 39, n. 2, p. 154-163, 2005.

REHEM, T. C. M. S. B.; TRAD, L. A. B. Home health care: subsidies for a primary care project in Brazil. Ciência e Saúde Coletiva, Rio de Janeiro, v. 10, p. 231-242, 2005.

RICCI, N. A.; KUBOTA, M. T.; CORDEIRO, R. C. Concordância de observações sobre a capacidade funcional de idosos em assistência domiciliar. Revista de Saúde Pública, São Paulo, v. 39, n. 4, p. 655-662, 2005.
RODRIGUES, M. R.; BRETAS, A. C. P. O significado da assistência domiciliária para os idosos e/ ou seu cuidador familiar matriculados em um Programa Público de Assistência Domiciliária ao Idoso. Acta Paulista Enfermagem, São Paulo, v. 15, n. 3, p. 60-71, 2002.

TAKAI, M. et al. The experience of burnout among home caregivers of patients with dementia: relations to depression and quality of life. Archives of Gerontology and Geriatrics, Shannon, v. 49, n. 1, p. e1-e5, 2009.

TANG, W. R. Hospice family caregivers quality of life. Journal of Clinical Nursing, Malden, v. 18, p. 63-72, 2009.

TRELHA, C. S. et al. Caracterização de idosos restritos ao domicílio e seus cuidadores. Revista Espaço para a Saúde, Londrina, v. 8, n. 1, p. 20-27, 2006.

TOMOMITSU, M. R. S. V.; LEMOS, N. D.; PERRACINI, M. R. Prevalência e fatores associados à fragilidade em cuidadores idosos. Revista Brasileira de Geriatria e Gerontologia, Rio de Janeiro, v. 4, p. 3-12, 2010.

VICKREY, B. G. et al. Development and preliminary evaluation of a quality of life measure targeted at dementia caregivers. Health and Quality of Live Outcomes, London, v. 7, p. 56-68, 2009.

WANDERLEY, M. B. Publicização do papel do cuidador domiciliar. São Paulo: IEE, PUC, 1998.

Recebido em: 15/12/2009

Reapresentado em: 22/08/2010

Aprovado em: 06/12/2010 